\title{
YAP and TAZ mediate steroid-induced alterations in the trabecular meshwork cytoskeleton in human trabecular meshwork cells
}

\author{
JIE PENG ${ }^{1,2}$, HAILIAN WANG $^{3}$, XIN WANG $^{2}$, MINGHAN SUN $^{4}$, SHAOPING DENG $^{3}$ and YI WANG ${ }^{2,5}$ \\ ${ }^{1}$ Department of Ophthalmology, Sichuan Academy of Medical Sciences and Sichuan Provincial People's Hospital, \\ Chengdu, Sichuan 610072; ${ }^{2}$ Department of Biomedical Engineering, Medical School of the University of \\ Electronic Science and Technology of China, Chengdu, Sichuan 610054; ${ }^{3}$ Institute of Organ Transplantation \\ and Departments of ${ }^{4}$ Gynecology and ${ }^{5}$ Pharmacy, Sichuan Academy of Medical Sciences and \\ Sichuan Provincial People's Hospital, Chengdu, Sichuan 610072, P.R. China
}

Received January 18, 2017; Accepted September 28, 2017

DOI: 10.3892/ijmm.2017.3207

\begin{abstract}
Primary open angle glaucoma is an important type of glaucoma as it is one of the most common causes of blindness. Previous studies have demonstrated that in glaucomatous patients, the human trabecular meshwork (HTM) is markedly stiffened. The purpose of the present study was to determine the regulatory role of Yes-associated protein (YAP) and transcriptional coactivator with PDZ-binding motif (TAZ) in HTM cells. Primary HTM cells were cultured with different concentrations of dexamethasone (DEX), and the expression levels of YAP and TAZ were evaluated using reverse transcription-quantitative polymerase chain reaction and western blotting. The results revealed that DEX increased the expression of YAP and TAZ in a dose-dependent manner. In addition, the western blot analysis of cytoskeleton-associated proteins revealed that the inhibition of YAP and/or TAZ using small interfering RNA resulted in the increased expression of collagen I, and decreased expression of fibronectin, laminin and collagen IV. The expression of $\beta$-catenin, a key protein in the Wnt pathway, was also observed to be regulated by YAP and TAZ. A 5-ethynyl-2'-deoxyuridine staining assay indicated that YAP and TAZ induced the proliferation of HTM cells. The investigation of cross-linked actin network formation by the HTM cells demonstrated that the knockdown of YAP and TAZ genes rescued HTM cells from cytoskeletal reorganization. Furthermore, functional evaluation of a HTM
\end{abstract}

Correspondence to: Dr Yi Wang, Department of Pharmacy, Sichuan Academy of Medical Sciences and Sichuan Provincial People's Hospital, 32 West Ring Road, Sichuan, Chengdu 610072, P.R. China

E-mail: w_yi@yahoo.com

Key words: glaucoma, human trabecular meshwork cells, dexamethasone, Yes-associated protein, transcriptional coactivator with PDZ-binding motif cell monolayer using a permeability assay demonstrated that the inhibition of YAP and TAZ attenuated the DEX-induced impairment of permeability. These findings suggest that YAP and TAZ play pivotal roles in the DEX-induced cytoskeletal changes of HTM cells, and reveal novel potential mechanisms for the development and progression of glaucoma.

\section{Introduction}

Glaucoma is the second most common cause of blindness worldwide, and $\sim 47 \%$ of the individuals with glaucoma are of Asian origin (1). Notably, primary open angle glaucoma (POAG) is one of the most prevalent types of glaucoma, and its main clinical manifestations are high intraocular pressure (IOP) and visual function damage (2). Although the mechanism of POAG development is unclear, the main pathological change is considered to be the reduced outflow of aqueous humor through the trabecular meshwork (TM), the major site of IOP regulation (3). A previous study has demonstrated that an increased amount of sheath-derived plaque material in the TM is associated with an increased severity of optic nerve damage in POAG (4). In addition, the TM from glaucomatous ocular tissue has been found to be stiffer than that from normal ocular tissue (5), indicating the significance of the morphological and biophysical changes of the TM in glaucoma. Therefore, primary cultured human trabecular meshwork (HTM) cells have been studied in vitro in order to investigate the pathogenic mechanism of POAG. Corticosteroids have long been recognized as a cause of glaucoma $(6,7)$. Thus, a commonly used in vitro model for glaucoma is the treatment of HTM cells with dexamethasone (DEX), a synthetic glucocorticoid (8-11).

The Hippo signaling pathway, first discovered in Drosophila, is a signaling pathway that controls organ size by promoting cell death and cell differentiation $(12,13)$ and inhibiting cell proliferation (14-17). Yes-associated protein (YAP) and transcriptional coactivator with PDZ-binding motif (TAZ) are two Yorkie homologs in the Hippo pathway that have been identified in mammals and are highly conserved (18). It has been reported that YAP and TAZ exist at all levels 
of the TM (19), and are influenced by the stiffness of the extracellular matrix (ECM) (20). Studies conducted by Raghunathan et al (19) demonstrated the expression of YAP and TAZ in HTM cells. In these studies, polyacrylamide hydrogels mimicking the normal $(5 \mathrm{kPa})$ and glaucomatous $(75 \mathrm{kPa})$ meshworks were used in the culture of HTM cells, and the researchers observed that YAP and TAZ mRNA expression levels were upregulated on the $75-\mathrm{kPa}$ hydrogels in comparison with the $5-\mathrm{kPa}$ hydrogels. However, to the best of our knowledge, no previous studies concerning the involvement of YAP and TAZ in corticosteroid-induced glaucoma have been reported. Therefore, the present study aimed to elucidate the roles of YAP and TAZ in DEX-induced glaucoma. The knockdown and overexpression of YAP and TAZ genes was conducted in order to investigate their roles in the regulation of DEX-induced glaucoma. In addition, the regulatory roles of YAP and TAZ in the cell proliferation and cytoskeletal structure of HTM cells were determined.

\section{Materials and methods}

Chemicals. DEX, fluorescein isothiocyanate (FITC)-labeled phalloidin (P5282), Triton X-100 (T9284), bovine serum albumin (BSA; V900933), Bradford reagent (B6916) and paraformaldehyde (PFA; 158127) were all purchased from Sigma-Aldrich (Merck KGaA, Darmstadt, Germany). Small interfering RNAs (siRNAs) against human YAP (sc-38637) and human TAZ (sc-38568), and scrambled siRNA (sc-37007) were purchased from Santa Cruz Biotechnology, Inc. (Dallas, TX, USA). Click-iT 5-ethynyl-2'-deoxyuridine (EdU) Alexa Fluor 488 Imaging kit (C10337), SYBR-Green PCR Master mix, NuPAGE 10\% Bis-Tris protein gel (NP0315BOX), NuPAGE transfer buffer (NP0006) and 4',6-diamidino2-phenylindole (DAPI; D1306) were purchased from Thermo Fisher Scientific, Inc. (Waltham, MA, USA). Polyvinylidene fluoride membranes were purchased from Pall Corporation (Port Washington, NY, USA).

Constitutively activated YAP (GST-YAP, Addgene plasmid 38105), TAZ (HA-TAZ, Addgene plasmid 32839) and empty vector (pcDNA3.1, Addgene plasmid 62803) were all purchased from Addgene, Inc. (Cambridge, MA, USA).

Primary antibodies to the following proteins were purchased from Santa Cruz Biotechnology, Inc.: YAP (sc-15407), TAZ (sc-48805), fibronectin (sc-53285), laminin $\alpha 1$ (sc-6016), $\beta$-catenin (sc-65480) and $\beta$-actin (sc-47778). Primary antibodies to collagen I (ab34710) and collagen IV (ab19808) were both purchased from Abcam (Cambridge, MA, USA). The secondary antibodies horseradish peroxidase (HRP)-conjugated goat anti rabbit IgG (cat. no. 11-035-003) and goat anti-mouse IgG (cat. no. 11-035-003) were both purchased from Jackson ImmunoResearch Laboratories, Inc. (West Grove, PA, USA).

Cell culture. HTM cells were purchased from Bolise Co., Ltd. (Shanghai, China). The cells were cultured in Dulbecco's modified Eagle's medium (DMEM)/F-12 containing $10 \%$ fetal bovine serum (FBS), $100 \mathrm{mg} / \mathrm{ml}$ streptomycin and $100 \mathrm{U} / \mathrm{ml}$ penicillin (all from Thermo Fisher Scientific, Inc.), and maintained at $37^{\circ} \mathrm{C}$ with $5 \% \mathrm{CO}_{2}$ in a humidified atmosphere. All studies were conducted using cells prior to the eighth passage.
Cell treatment, proliferation assay and morphological analysis. HTMC cells were treated with PBS or DEX $\left(1 \times 10^{-4}\right.$, $1 \times 10^{-5}, 1 \times 10^{-6}$ and $\left.1 \times 10^{-7} \mathrm{M}\right)$ at $37^{\circ} \mathrm{C}$ for $48 \mathrm{~h}$. Cell proliferation was analyzed using a Click-iT EdU cell proliferation assay kit according to the manufacturer's instructions. In brief, $3 \times 10^{5}$ HTM cells were plated on coverslips and incubated with $10 \mu \mathrm{M}$ EdU solution at $37^{\circ} \mathrm{C}$ for $16 \mathrm{~h}$. The cells were then fixed with $4 \%$ buffered PFA and incubated with $0.5 \mathrm{ml}$ Click-iT reaction mixture for $30 \mathrm{~min}$ at room temperature. The coverslides were examined using a Nikon Eclipse 80i microscope system (Nikon, Tokyo, Japan). DAPI was used as a nuclear counterstain (blue) at room temperature for $5 \mathrm{~min}$. EdU-positive cells (green) in 15-20 randomly selected fields were manually counted and the proportion of all cells that were EdU-positive was determined.

For the immunofluorescence analysis of cell morphology, $3 \times 10^{5}$ HTM cells plated on coverslips were fixed with $4 \%$ PFA and permeabilized with Triton X-100. Following the removal of the remaining Triton X-100, the cells were incubated with $5 \mu \mathrm{g} / \mathrm{ml}$ FITC-labeled phalloidin for $1 \mathrm{~h}$. Nuclear staining of the cells was conducted by staining with DAPI at room temperature for $5 \mathrm{~min}$. The coverslides were examined using the Nikon Eclipse 80i microscope system.

Transfection and gene silencing. HTM cells were plated in 60-mm dishes with DMEM/F-12 containing 10\% FBS and $1 \%$ penicillin-streptomycin at a density of $1 \times 10^{6}$ cells/dish. The plated HTM cells were continuously cultured in DMEM/F-12 without $10 \%$ FBS and without $1 \%$ penicillin-streptomycin for a further $2 \mathrm{~h}$. The YAP, TAZ and empty plasmids were mixed with 25X diluent Lipofectamine LTX with PLUS reagent, and incubated for $20 \mathrm{~min}$ at room temperature to allow plasmid-Lipofectamine LTX complexation. Transfections were then conducted in OptiMEM reduced-serum medium (cat. no. 31985070; Thermo Fisher Scientific, Inc.) containing $1 \mathrm{mg} / \mathrm{ml}$ plasmid DNA, $0.1 \%$ PLUS reagent and $0.3 \%$ Lipofectamine LTX transfection reagent, where HTM cells were cultured in the medium with plasmid in $60-\mathrm{mm}$ dishes for $48 \mathrm{~h}$ (21). Finally, the transfection efficiency was detected using western blot analysis. For the knockdown of YAP and TAZ genes, HTM cells were transfected with YAP siRNA, TAZ siRNA or scrambled siRNA at $33 \mathrm{nM}$ using DharmaFect transfection reagent with SMARTpool ON-TARGET siRNA (GE Healthcare Dharmacon, Inc., Lafayette, CO, USA) according to the manufacturer's instructions (22). The transfected cells were used for subsequent experiments $24 \mathrm{~h}$ later.

Western blot analysis. The cells were lysed using RIPA lysis buffer (Beyotime Institute of Biotechnology, Suzhou, China) and the protein lysates were centrifuged at $13,400 \mathrm{x} \mathrm{g}$ for $30 \mathrm{~min}$. The supernatants were collected and quantified using the Bradford protein assay method with BSA as the standard. The protein was then denatured by heating to $95^{\circ} \mathrm{C}$ for $5 \mathrm{~min}$. Approximately $20 \mu \mathrm{g}$ protein was loaded into each well of the precast NuPAGE 10\% Bis-Tris protein gel. Following electrophoresis, the proteins were transferred onto a polyvinylidene fluoride membrane using the NuPAGE transfer buffer. The membranes were blocked with 5\% BSA in TBST at room temperature for $1 \mathrm{~h}$, and then incubated overnight at $4^{\circ} \mathrm{C}$ with primary antibodies at the dilutions recommended 
by the manufacturer (1:500). The blots were then incubated with HRP-conjugated IgG secondary antibodies (1:5,000 dilutions) for $1 \mathrm{~h}$ at $37^{\circ} \mathrm{C}$. Bands were detected using Immobilon Western Chemiluminescent HRP Substrate (WBKLS0500; EMD Millipore, Billerica, MA, USA) and imaged using an ImageQuant 350 system (GE Healthcare Life Sciences, Piscataway, NJ, USA) (23).

Reverse transcription-quantitative polymerase chain reaction (RT-qPCR). RNA was extracted from the HTM cells using an RNeasy Isolation kit according to the manufacturer's instructions (Qiagen, Inc., Valencia, CA, USA), and then reverse transcribed. The reaction mixture comprised $1 \mu \mathrm{g}$ total RNA, $1 \mu$ l random primer (50 $\mu \mathrm{mol} / 1$, cat. no. 48190011 ; Thermo Fisher Scientific, Inc.), $1 \mathrm{X}$ reverse transcription buffer and 10 units reverse transcriptase (A5003; Promega, Madison, WI, USA) in a total volume of $20 \mu \mathrm{l}$. The RNA and primer were heated to $72^{\circ} \mathrm{C}$ and slowly cooled prior to reverse transcription at $42^{\circ} \mathrm{C}$ for $1 \mathrm{~h}$ (24). When cooled to room temperature, the reaction was diluted to $100 \mu \mathrm{l}$ with RNase-free water. qPCR, was carried out with SYBR-Green PCR Master mix in a total reaction volume of $20 \mu \mathrm{l}$ using the following amplification steps: Initial denaturation at $95^{\circ} \mathrm{C}$ for $10 \mathrm{~min}$; followed by 40 cycles of denaturation at $95^{\circ} \mathrm{C}$ for $15 \mathrm{sec}$; and then elongation at $55^{\circ} \mathrm{C}$ for $30 \mathrm{sec}$. The expression levels were normalized to those of the internal standard 18S rRNA. The following primer sequences were used: YAP sense, 5'-ACCCACAGCTCAGCATCTTCG-3' and antisense, 5'-TGGCTTGTTCCCATCCATCAG-3'; TAZ sense, 5'-GTCACCAACAGTAGCTCAGATC-3' and antisense, 5'-AGTGATTACAGCCAGGTTAGAAAG-3'; 18S rRNA sense, 5'-GGGCATTCGTATTTCATAGTCAGAG-3' and antisense, 5'-CGGTTCTTGATTAATGAAAACATCCT-3'. The experiment was performed in triplicate. The YAP and TAZ mRNA expression levels of the DEX-treated groups (treated with $10 \mu \mathrm{M}$ dexamethasone at $37^{\circ} \mathrm{C}$ for $48 \mathrm{~h}$ ) were normalized relative to those of the DMSO (DEX-free) group, which was assigned a value of 1.0. In order to control for slight variations in the amount of RNA loaded for PCR, the difference in cycle threshold $(\Delta \mathrm{Cq})$ between the gene of interest and the average cycle threshold $(\mathrm{Cq})$ of the house keeping gene 18S (also loaded in triplicate wells) were calculated. By calculating the difference in $\triangle \mathrm{Cq}$ between the DEX-treated group and the control group, the experimental data was normalized to the control data using the $2^{-\Delta \Delta \mathrm{Cq}}$ method (25).

Permeability assay. The in vitro permeability assay was performed as previously reported (26). Briefly, HTM cells were plated onto the cell culture inserts of Transwell plates (cat. no. 353104, BD Falcon; BD Biosciences, Franklin Lakes, NJ, USA). When the cells reached subconfluence, they were transfected with the aforementioned siRNA or plasmids. At 3 days after transfection, the cells were subjected to an in vitro permeability assay. On the day of the assay, the medium in the upper and lower chambers was replaced with fresh DMEM, and 150 $\mu$ l FITC-Dextran (1:30 dilution, D1845; Thermo Fisher Scientific, Inc.) was added to the medium in the upper chamber of each insert. After $5 \mathrm{~min}$ at room temperature, $100 \mu \mathrm{l}$ solution from the lower chamber was transferred to a 96-well plate. The plate was read using an Envision 2103 Multilabel Plate Reader (PerkinElmer, Inc., Waltham, MA, USA) at an excitation wavelength of $480 \mathrm{~nm}$ and emission wavelength of $530 \mathrm{~nm}$, with a bandwidth of 10 .

Statistical analysis. All data are presented as mean \pm standard error of the mean from at least three independent experiments. Statistical significance was determined by one-way analysis of variance followed by Bonferroni correction when three or more groups were compared, and by Student's t-test when two groups were compared. $\mathrm{P}<0.05$ was considered to indicate a statistically significant difference.

\section{Results}

DEX induces elevated YAP and TAZ expression in HTM cells. A previous study has demonstrated a marked stiffness of the HTM in glaucomatous patients (5). In addition, the HTM substratum stiffness closely correlates with the expression and activity of the YAP and TAZ proteins (27). Thus, it was hypothesized that DEX-induced substratum stiffness is accompanied by elevated expression levels of YAP and TAZ. Staining of the HTM cells with the F-actin probe FITC-phalloidin, as shown in Fig. 1A-E, indicates that DEX altered the F-actin architecture in a concentration-dependent manner, and promoted the formation of a cross-linked actin network (CLAN) in the HTM cells (10). To determine whether YAP and TAZ transcription was associated with the DEX induced-CLAN formation, RT-qPCR was performed. It was observed that $1 \times 10^{-5} \mathrm{M}$ DEX induced a significant increase in the transcription level of the YAP gene (Fig. 1F). In addition, the transcription level of the TAZ gene was also significantly elevated in the presence of $1 \times 10^{-5} \mathrm{M}$ DEX (Fig. 1G). These results suggest an association of the DEX-induced cytoskeletal changes in the cultured HTM cells with the increased transcription levels of YAP and TAZ. To further validate the correlation of the expression levels of YAP and TAZ with the DEX-induced cytoskeletal reorganization, western blotting was performed. As shown in Fig. 1H, the expression levels of the YAP and TAZ proteins in the HTM cells were observed to increase as the concentration of DEX increased. These data indicate that DEX has a dose-dependent association with the transcription and expression levels of YAP and TAZ.

YAP and TAZ regulate actin-associated proteins. Previous studies have demonstrated that in response to DEX treatment, several actins and actin-associated proteins are involved in the development of a CLAN (28). Furthermore, among those proteins, fibronectin (29) and laminin (30) have been shown to be increased in DEX-treated HTM cells. Therefore, in order to further elucidate the effects of YAP and TAZ on actin-associated proteins, YAP and TAZ genes were knocked down using siRNA (Fig. 2A), and the expression levels of fibronectin and laminin were measured. As shown by the western blotting results in Fig. 2B, DEX markedly increased the expression levels of fibronectin and laminin. However, the elevated expression levels were attenuated by the addition of YAP and TAZ siRNA. The expression of ECM-associated molecules, including collagen types I and IV, is known to be associated with DEX-induced CLAN formation $(31,32)$. Thus, the expression levels of types I and IV collagen were 
A

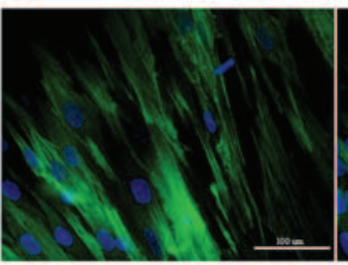

B

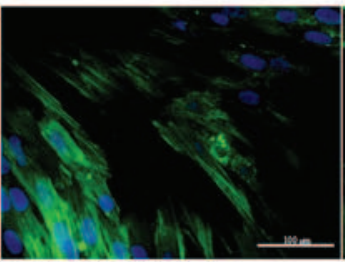

C

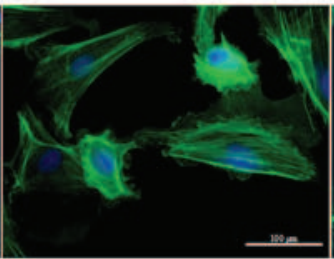

D

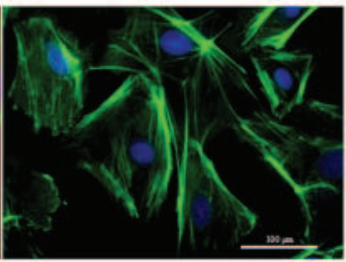

E

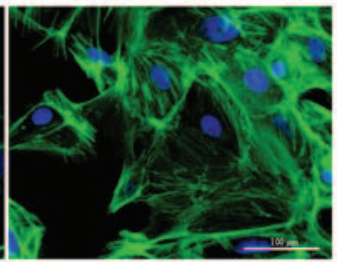

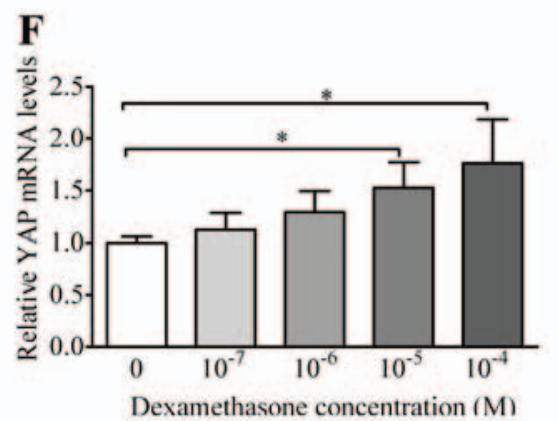

G

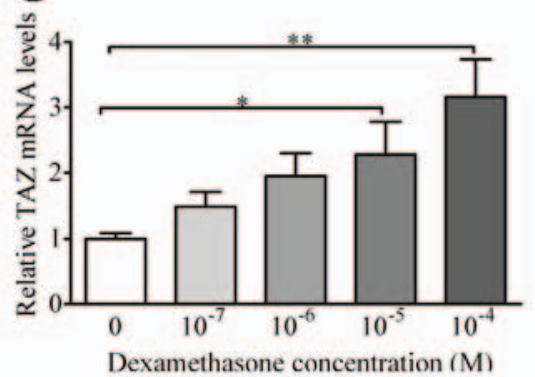

H

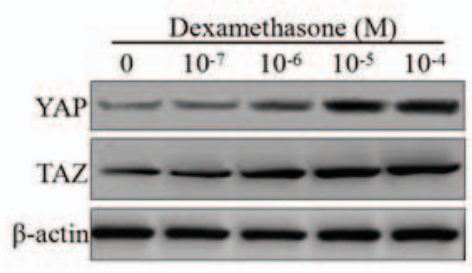

Figure 1. DEX increases the expression of YAP and TAZ. (A-E) Observation of DEX-induced cross-linked actin network formation by HTM cells. The cells were treated with (A) DMSO, (B) $1 \times 10^{-7} \mathrm{DEX}$, (C) $1 \times 10^{-6} \mathrm{DEX}$, (D) $1 \times 10^{-5} \mathrm{DEX}$ and (E) $1 \times 10^{-4} \mathrm{DEX}$. Green, F-actin stain; blue, nuclear stain (DAPI). Scale bar, $100 \mu \mathrm{m}$. (F) YAP mRNA levels and (G) TAZ mRNA levels in HTM cells treated with different concentrations of DEX. (H) Western blot analysis of YAP and TAZ proteins in the DEX-treated HTM cells. Protein bands shown are representative of three independent experiments with similar results. Data are presented as the mean \pm standard error of the mean from three independent experiments. ${ }^{*} \mathrm{P}<0.05$ and ${ }^{* *} \mathrm{P}<0.01$. DEX, dexamethasone; YAP, Yes-associated protein; TAZ, transcriptional coactivator with PDZ-binding motif; HTM, human trabecular meshwork.
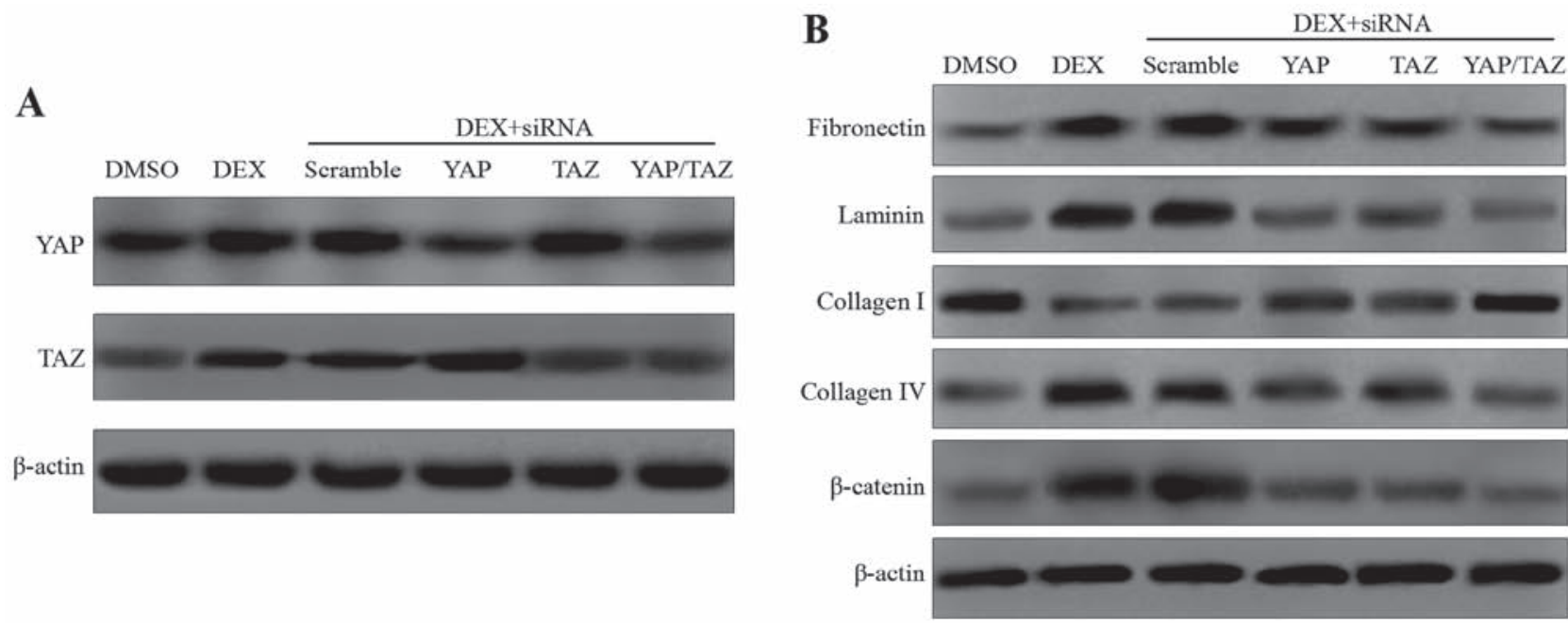

Figure 2. Knockdown of YAP and TAZ mRNA results in the inhibition of actin-related proteins in DEX-stimulated HTM cells. (A) Western blot analysis demonstrating the efficiency of YAP siRNA knockdown and/or TAZ siRNA knockdown. (B) Western blot analysis of fibronectin, laminin, collagen I, collagen IV and $\beta$-catenin following transfection with YAP siRNA and/or TAZ siRNA. Protein bands shown are representative of three independent experiments with similar results. YAP, Yes-associated protein; TAZ, transcriptional coactivator with PDZ-binding motif; DEX, dexamethasone; HTM, human trabecular meshwork; siRNA, small interfering RNA.

determined in the present study. Increased expression levels of type I collagen and decreased expression levels of type IV collagen were observed in the HTM cells treated with DEX plus YAP and/or TAZ siRNA concurrently compared with those treated with DEX alone (Fig. 2B). In addition, a previous study by the present research team demonstrated that the expression of $\beta$-catenin was induced by DEX in cultured HTM cells (33). The present study confirmed this, and revealed that $\beta$-catenin expression was decreased in the DEX-treated cells transfected with YAP and/or TAZ siRNA compared with that in the DEX control.
To further substantiate the association of YAP and TAZ with the proteins shown in Fig. 2B, the HTM cells were cotransfected with YAP and/or TAZ expression plasmids (Fig. 3A). The western blotting results presented in Fig. 3B indicate that the expression levels of fibronectin and laminin were increased in the presence of YAP and TAZ overexpression, and the expression was comparable to that in the DEX-treated cells. In addition, the expression of type I collagen was inhibited in the YAP and TAZ overexpression groups, whereas the expression of type IV collagen was clearly increased. Increased expression of $\beta$-catenin was also observed in the YAP and TAZ overexpressing 
A

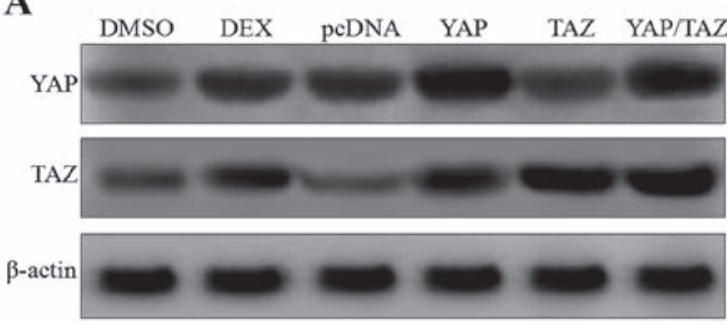

B

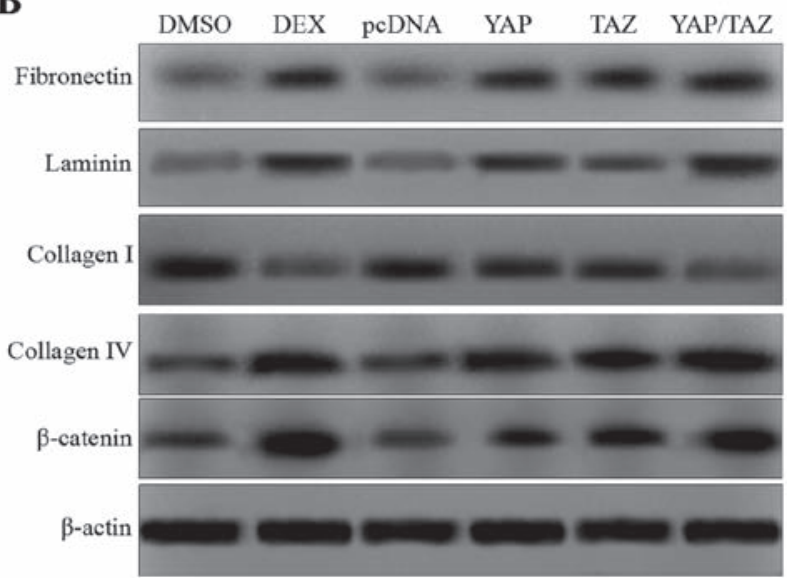

Figure 3. Overexpression of YAP and TAZ mRNA results in the activation of actin-related proteins in HTM cells. (A) Western blot analysis demonstrating the efficiency of YAP overexpression and/or TAZ overexpression. (B) Western blot analysis of fibronectin, laminin, collagen I, collagen IV and $\beta$-catenin following transfection with plasmids. Protein bands shown are representative of three independent experiments with similar results. YAP, Yes-associated protein; TAZ, transcriptional coactivator with PDZ-binding motif; HTM, human trabecular meshwork; DEX, dexamethasone; pcDNA, empty vector.

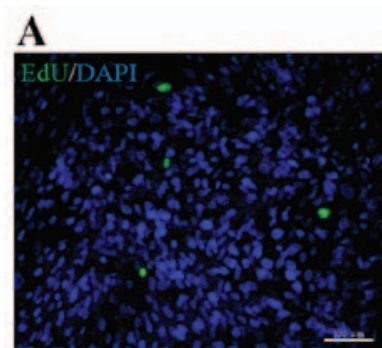

Scramble siRNA

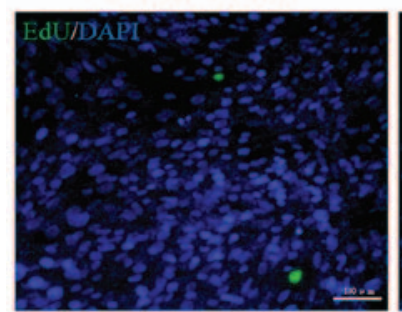

TAZ siRNA

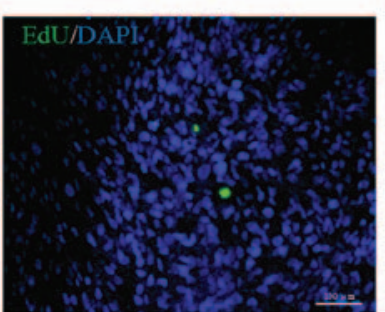

YAP siRNA

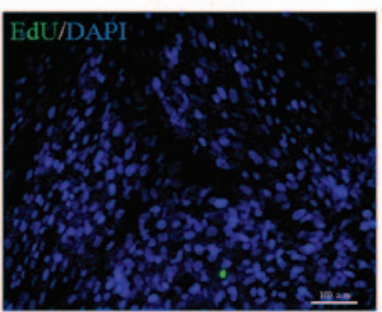

YAP/TAZ SIRNA

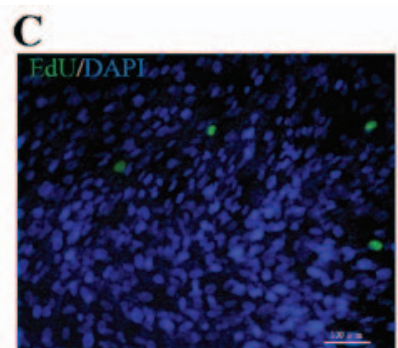

peDNA3.1

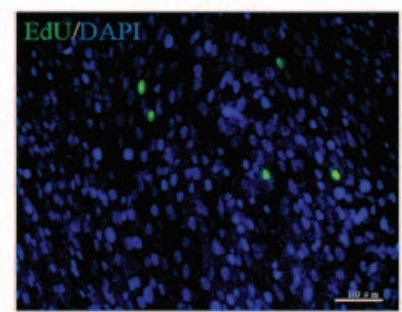

TAZ

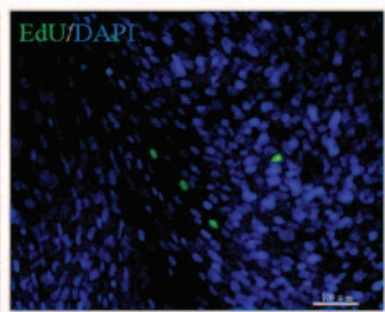

YAP

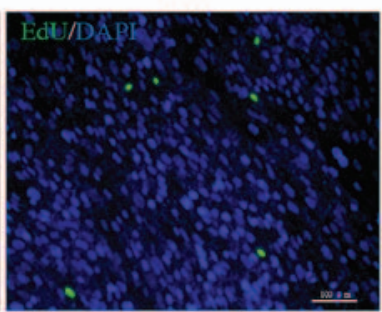

YAP/TAZ
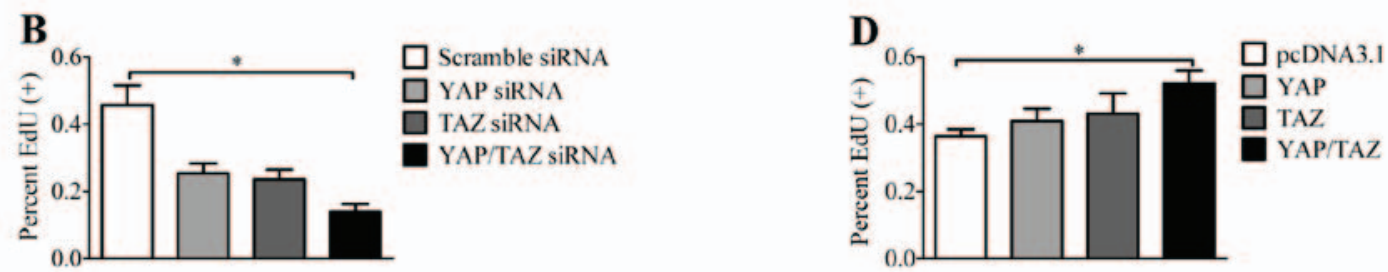

Figure 4. YAP and TAZ induce the proliferation of HTM cells. (A) EdU fluorescent staining of HTM cells following transfection with YAP siRNA and/or TAZ siRNA and (B) quantified results. (C) EdU fluorescent staining of HTM cells following transfection with YAP and/or TAZ expression plasmids and (D) quantified results. (A and C) Blue staining indicates the DAPI-labeled cell nuclei, and green staining indicates the EdU-labeled nuclei of proliferating cells. Magnification, x100. (B and D) Quantification was performed by manually counting the EdU-positive HTM cells, and calculating the proliferation percentages. Data are the mean \pm standard error of the mean from three independent experiments. ${ }^{*} \mathrm{P}<0.05$. YAP, Yes-associated protein; TAZ, transcriptional coactivator with PDZ-binding motif; HTM, human trabecular meshwork; siRNA, small interfering RNA; EdU, 5-ethynyl-2'-deoxyuridine.

cells. These data demonstrate that YAP and TAZ regulate actinassociated proteins and the expression of $\beta$-catenin.

YAP and TAZ induce the proliferation of HTM cells. The Hippo/Yap pathway is a well-conserved signaling cascade that regulates cell proliferation and differentiation, and controls organ size $(34,35)$. In the present study, the proliferation of HTM cells was investigated by labeling the cells with EdU to fluorescently stain the replicating cells. Notably, the fluorescence images indicate that the cells in which the YAP and TAZ genes were both knocked down possessed significantly reduced proliferation capability (Fig. 4A and B), 
A

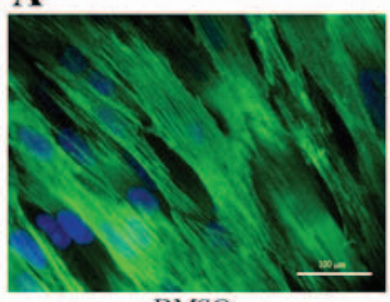

DMSO

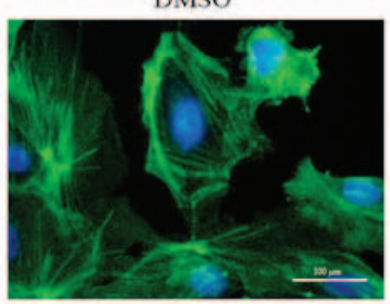

DEX + Seramble siRNA

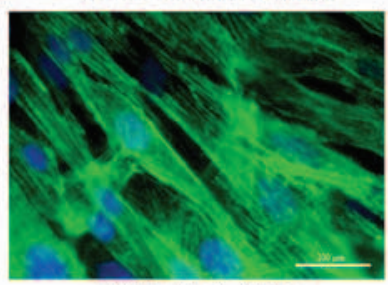

DEX + TAZ siRNA

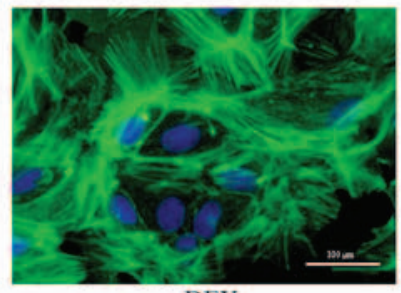

DEX

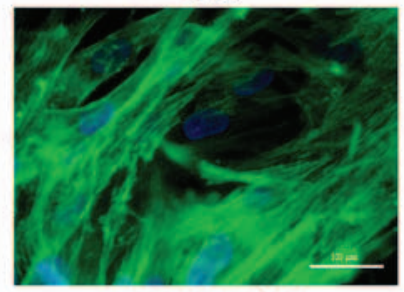

DEX+YAP siRNA

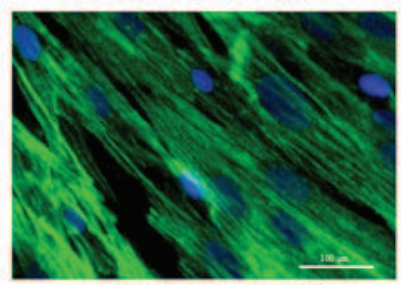

DEX + YAP/TAZ SiRNA

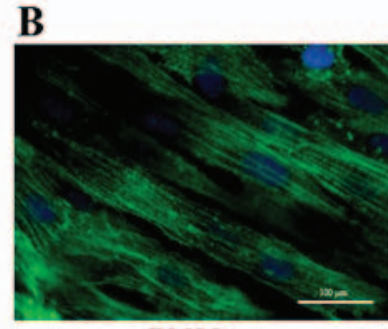

DMSO

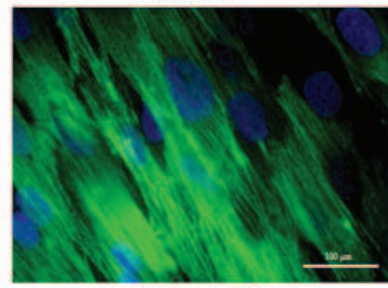

DMSO'peDNA3.1

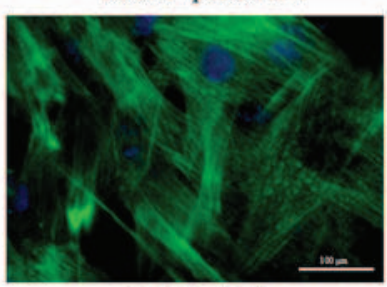

DMSO+TAZ

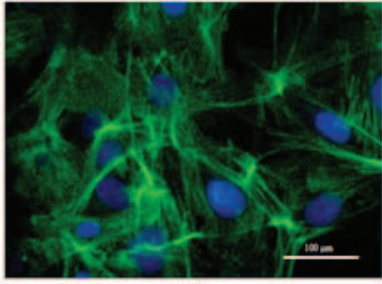

DEX

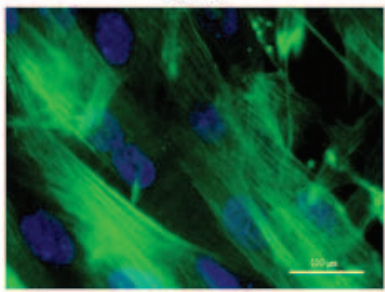

DMSO+YAP

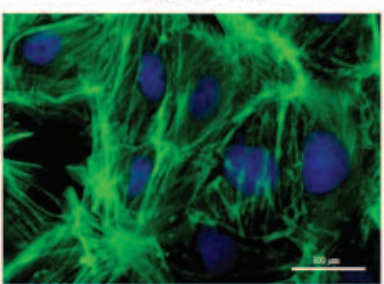

DMSO+YAP/TAZ

Figure 5. Inhibition of YAP and TAZ rescues HTM cells from cross-linked actin network formation. (A) Immunofluorescent staining of the cytoskeleton of HTM cells in the presence of YAP siRNA and/or TAZ siRNA. (B) Immunofluorescent staining of the cytoskeleton of HTM cells in the presence of YAP and/or TAZ plasmids. Blue staining indicates the DAPI-labeled cell nuclei, and green staining indicates cytoskeletal staining with fluorescein isothiocyanate-labeled phalloidin. Magnification, x400. YAP, Yes-associated protein; TAZ, transcriptional coactivator with PDZ-binding motif; HTM, human trabecular meshwork; siRNA, small interfering RNA; DEX, dexamethasone; pcDNA, empty vector.

indicating that YAP and TAZ may have a pivotal role in the regulation of HTM cell proliferation. By contrast, as depicted in Fig. 4C and D, when expression vectors for YAP and TAZ were employed, the HTM cells cotransfected with YAP and TAZ plasmids exhibited significantly increased proliferation ability. These results indicate the involvement of YAP and TAZ in the regulation of the proliferation of HTM cells.

YAP and TAZ regulate CLAN formation in HTM cells. Since CLAN formation in glaucomatous and DEX-treated HTM cells has been indicated to contribute to reduced outflow facility (36), CLAN formation in the presence of DEX, YAP siRNA and/or TAZ siRNA was investigated in the present study. From the images presented in Fig. 5A, it is clear that DEX-treated HTM cells formed unusual geodesic dome-like cross-linked actin networks. The majority of these cells exhibited a CLAN, which was composed of a series of interconnected F-actin bundles that radiated outward from central vertices in a geodesic network. However, YAP siRNA and TAZ siRNA appeared to markedly alter overall cell spreading, and induced reorganization of the actin skeleton. Thus, is appears that CLAN formation in the HTM cells cotransfected with YAP siRNA and TAZ siRNA was attenuated. The actin cytoskeleton and actin microfilament patterns were comparable to those of the DMSO-treated control cells. To further evaluate whether YAP and TAZ contribute to rearrangement of the cytoskeleton in HTM cells, YAP and/or TAZ overexpression plasmids were transfected into the HTM cells. As shown in Fig. 5B, the overexpression of YAP and TAZ induced CLAN formation with polygonal actin arrangements in the HTM cells. The actin filaments were dispersed around the cell, with a few microfilaments in the center, and were similar in appearance to those of the DEX-treated control cells. These observations suggest that YAP and TAZ play a key role in CLAN formation in HTM cells.

YAP and TAZ modulate the aqueous humor outflow of HTM cells. It has been suggested that DEX-induced CLAN formation in HTM cells decreases the overall contractility of the tissue by causing the cells to become more rigid and unable to change shape (10). Therefore, the permeability of DEX-treated monolayer HTM cells may be markedly impaired. In order to further investigate the hypothesis that YAP and TAZ regulate the function of HTM cells, a permeability assay was performed. The DEX-treated monolayer HTM cells exhibited impaired permeability, which is consistent with previous studies $(26,37)$. However, when YAP and TAZ expression was downregulated with a combination of YAP and TAZ siRNA, the permeability was increased compared with that in the cells treated with DEX alone and those treated with DEX plus scramble siRNA (Fig. 6A). In addition, cells transfected with a combination of YAP and TAZ expression plasmids exhibited significantly decreased permeability compared with the cells treated with DMSO and transfected with an empty plasmid (Fig. 6B). This observation indicates that YAP and TAZ regulate aqueous outflow and, thereby alter the function of HTM cells. 
A
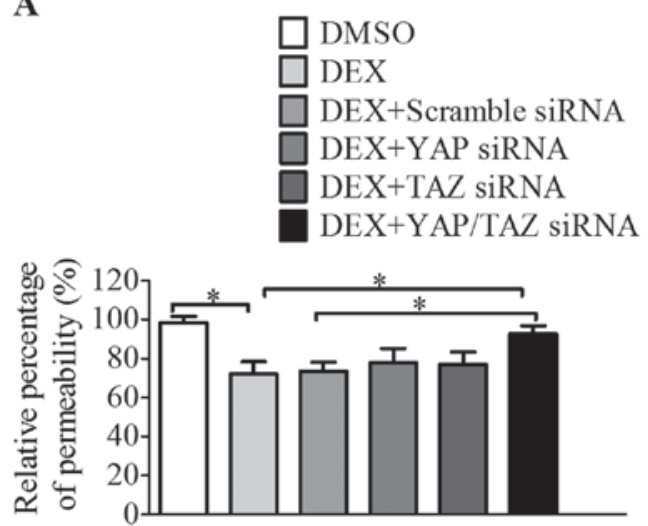

B
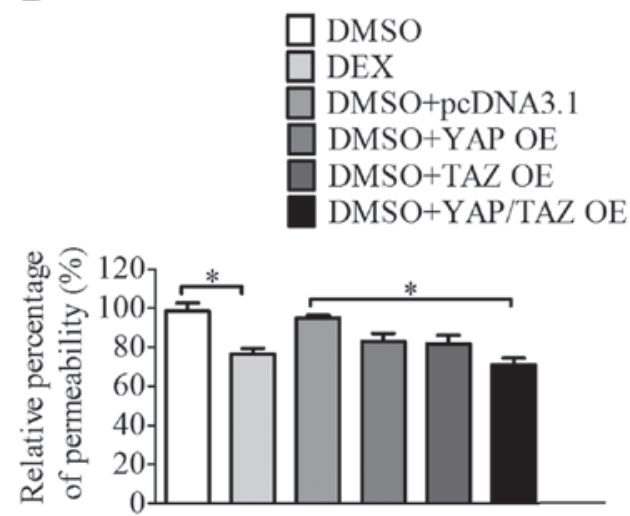

Figure 6. YAP and TAZ modulate aqueous humor outflow via the inhibition of permeability. Relative permeability percentages of HTM cell monolayers transfected with (A) YAP siRNA and/or TAZ siRNA and (B) YAP and/or TAZ expression plasmids. Data are the mean \pm standard error of the mean from three independent experiments. ${ }^{*} \mathrm{P}<0.05$. YAP, Yes-associated protein; TAZ, transcriptional coactivator with PDZ-binding motif; HTM, human trabecular meshwork; siRNA, small interfering RNA; DEX, dexamethasone; pcDNA, empty vector; OE, overexpression.

\section{Discussion}

POAG is a common cause of blindness (1). Although the exact cause of POAG is not yet clearly understood, the main abnormal changes associated with this condition appear to affect the TM (3). TM cells are capable of the phagocytosis, migration, synthesis and secretion of ECM components, and the transduction of signals between ECMs. Dysfunction of the TM and the lining cells of Schlemm's canal, as well as structural abnormalities of the ECM are the main causes of resistance to the outflow of aqueous humor. Changes in ECM components cause narrowing of the outflow tract in adjacent areas, thereby increasing resistance to the outflow of aqueous humor and elevating the IOP (38). In the present study, the signal transduction of YAP and TAZ in HTM cells, and its regulatory role in the cytoskeletal arrangement of HTM cells were investigated.

The symptoms of POAG include increased resistance to the outflow of aqueous humor, increased TM hardness, hardening and degeneration of the trabecular tissue, reduced mesh density, irregularity or injury of the trabecular plate layer, increased endothelial cell density, the degeneration of collagen or elastic fibers, and the narrowing of spaces in the TM (5). These changes in TM properties are indicative of a biophysical mechanism for the regulation of IOP and glaucoma (39-43). However, the molecular mechanism by which the biophysical properties affect cell behaviour is not yet completely understood. YAP and TAZ have been identified as mechanical signaling factors, which are affected by ECM hardness (20). YAP and TAZ are the main effectors of the classical Hippo pathway in the regulation of organ size and tissue topology; however, they are considered to exert actions beyond those of the classical pathway due to their dynamic transduction effects as nuclear transcription factors in mechanical signaling (44). As mechanotransducers of the extracellular-microenvironment and coactivators of transcription, YAP and TAZ have been shown to be upregulated by the elastic hardness of the TM in glaucoma (19). In addition, previous studies have demonstrated that YAP serves important roles in the regulation of cell proliferation and apoptosis, the control of organ size, the contact inhibition of cells and tumorigenesis (13-17,44).

Glucocorticoids have been shown to cause progressive changes in the organization of the microfilaments in TM cells $(31,45)$. Therefore, in the present study, the effects of DEX on HTM cells were investigated. It was observed that as the concentration of DEX increased, the expression levels of YAP also significantly increased, and the cytoskeleton was damaged. TAZ and YAP genes are both conjugated with 14-3-3 $\sigma$ protein, and have the same transcription activation function (46). The similarity of the TAZ and YAP genes was reflected in the dose response data for DEX, which demonstrated that the expression levels of TAZ were also elevated as the concentration of DEX increased. It has been reported that in HTM cells cultured under high pressure, the expression levels of YAP and TAZ greatly exceed than those of HTM cells cultured at normal pressure (19). Their study demonstrated that numerous proteins are involved in the dynamic induction and transduction process, and identified that YAP and TAZ are dynamic transduction and transcription factors. YAP and TAZ have been confirmed to exist in all layers of the TM, including adjacent tissue (14), which is considered to be important in the generation of resistance to the outflow of aqueous humor. Therefore, the further exploration of YAP and TAZ genes in glaucoma is merited.

The fluorescence microscopic analysis conducted in the present study demonstrated that the knockdown of YAP and TAZ rescued HTM cells from the morphological changes induced by DEX. Furthermore, it revealed that the overexpression of YAP and TAZ contributed to the reorganization of HTM cells to form geodesic-dome-like polygonal lattices. Since reorganization of the TM cytoskeleton alters cell function $(8,47)$, the normalization of HTM cell morphology by silencing the expression of YAP and TAZ was observed to restore the functionality of the HTM cells in the presence of DEX in the cell permeability assay.

The glucocorticoid treatment of cultured TM cells has been reported to inhibit TM cell migration and proliferation (45). Therefore, the present study investigated the effect of YAP and TAZ knockdown on the proliferation of HTM cells. 
It has been reported that $\beta$-catenin binds with E-cadherin to bridge between the cytoplasmic domain of cadherin and the actin cytoskeleton, thus realizing a connection between cells and affecting the adhesion and motility of cells (48). The Wnt signaling pathway is considered a new target for intervention in the treatment of glaucoma, and the $\mathrm{Wnt} / \beta$-catenin signaling pathway has been demonstrated to be modulated by YAP and TAZ, with TAZ exhibiting antagonistic effects $(49,50)$. In the present study, it was demonstrated that the knockdown of YAP and TAZ decreased the expression levels of $\beta$-catenin, whereas YAP and TAZ overexpression increased them. In addition, the present study indicated that YAP and TAZ overexpression stimulated cell proliferation.

The present study on the mechanisms underlying the effects of YAP and TAZ in HTM cells indicates that YAP and TAZ serve critical roles in glaucoma. The expression of YAP and TAZ in the cultured TM cells of normal human eyes was demonstrated by western blotting and RT-qPCR. Notably, when the HTM cells were treated with DEX, the expression levels of YAP and TAZ were elevated, indicating the involvement of YAP and TAZ in the pathogenesis of glaucoma. The treatment of cultured TM cells with DEX is a classical model with which to simulate the state of TM cells in glucocorticoid-induced glaucoma. In the present study, the treatment of TM cells with DEX was demonstrated to markedly increase the expression levels of fibronectin, laminin and collagen IV in the ECM, reduce the expression of collagen I, induce the restructuring of actin microfilaments, promote the formation of classical cross-linked actin networks, and increase the expression of $\beta$-catenin. These changes are likely to result in increased TM hardness, restructuring of the TM and dynamic changes in the microenvironment.

In conclusion, in the present study, the effects of the knockdown and overexpression of YAP and TAZ on the expression levels of fibronectin, laminin, collagen I, collagen IV and $\beta$-catenin were analyzed, and the effects of YAP and TAZ on the ECM and the actin microfilaments and cytoskeletons of DEX-treated HTM cells were observed. The findings improve our understanding of the involvement of YAP and TAZ in glaucoma pathogenesis, and suggest new mechanisms for the development and progression of glaucoma.

\section{Acknowledgements}

This study was supported by the National Science Foundation of China (grant no. 81300768), the Sichuan Science Foundation (grant no. 2015JY0183), the Sichuan Health and Family Planning Commission (grant no. 16ZD025), and by funding from Sichuan Provincial People's Hospital and the Sichuan Scientific Research Foundation of Returned Overseas Chinese Scholars for Dr. Yi Wang.

\section{References}

1. Quigley HA and Broman AT: The number of people with glaucoma worldwide in 2010 and 2020. Br J Ophthalmol 90: 262-267, 2006

2. Gordon MO, Beiser JA, Brandt JD, Heuer DK, Higginbotham EJ, Johnson CA, Keltner JL, Miller JP, Parrish RK 2nd, Wilson MR et al: The Ocular Hypertension Treatment Study: baseline factors that predict the onset of primary open-angle glaucoma. Arch Ophthalmol 120: 714-720,2002.
3. Johnson M: 'What controls aqueous humour outflow resistance?'. Exp Eye Res 82: 545-557, 2006.

4. Gottanka J, Johnson DH, Martus P and Lütjen-Drecoll E: Severity of optic nerve damage in eyes with POAG is correlated with changes in the trabecular meshwork. J Glaucoma 6: 123-132, 1997.

5. Last JA, Pan T, Ding Y, Reilly CM, Keller K, Acott TS, Fautsch MP, Murphy CJ and Russell P: Elastic modulus determination of normal and glaucomatous human trabecular meshwork. Invest Ophthalmol Vis Sci 52: 2147-2152, 2011.

6. Kersey JP and Broadway DC: Corticosteroid-induced glaucoma: a review of the literature. Eye (Lond) 20: 407-416, 2006.

7. Stokes J, Walker BR, Campbell JC, Seckl JR, O'Brien C and Andrew R: Altered peripheral sensitivity to glucocorticoids in primary open-angle glaucoma. Invest Ophthalmol Vis Sci 44: 5163-5167, 2003.

8. Zhuo YH, He Y, Leung KW, Hou F, Li YQ, Chai F and Ge J: Dexamethasone disrupts intercellular junction formation and cytoskeleton organization in human trabecular meshwork cells. Mol Vis 16: 61-71, 2010.

9. Knepper PA, Collins JA and Frederick R: Effects of dexamethasone, progesterone, and testosterone on IOP and GAGs in the rabbit eye. Invest Ophthalmol Vis Sci 26: 1093-1100, 1985.

10. Clark AF, Brotchie D, Read AT, Hellberg P, English-Wright S, Pang IH, Ethier CR and Grierson I: Dexamethasone alters F-actin architecture and promotes cross-linked actin network formation in human trabecular meshwork tissue. Cell Motil Cytoskeleton 60: 83-95, 2005.

11. Clark AF, Wilson K, McCartney MD, Miggans ST, Kunkle M and Howe W: Glucocorticoid-induced formation of cross-linked actin networks in cultured human trabecular meshwork cells. Invest Ophthalmol Vis Sci 35: 281-294, 1994.

12. Kango-Singh M and Singh A: Regulation of organ size: insights from the Drosophila Hippo signaling pathway. Dev Dyn 238: 1627-1637, 2009 .

13. Saucedo LJ and Edgar BA: Filling out the Hippo pathway. Nat Rev Mol Cell Biol 8: 613-621, 2007.

14. Buttitta LA and Edgar BA: How size is controlled: from Hippos to Yorkies. Nat Cell Biol 9: 1225-1227, 2007.

15. Pan D: Hippo signaling in organ size control. Genes Dev 21: 886-897, 2007.

16. Zhao B, Lei QY and Guan KL: The Hippo-YAP pathway: new connections between regulation of organ size and cancer. Curr Opin Cell Biol 20: 638-646, 2008.

17. Yu FX and Guan KL: The Hippo pathway: regulators and regulations. Genes Dev 27: 355-371, 2013.

18. Wang K, Degerny C, Xu M and Yang XJ: YAP, TAZ, and Yorkie: a conserved family of signal-responsive transcriptional coregulators in animal development and human disease. Biochem Cell Biol 87: 77-91, 2009.

19. Raghunathan VK, Morgan JT, Dreier B, Reilly CM, Thomasy SM, Wood JA, Ly I, Tuyen BC, Hughbanks M, Murphy CJ, et al: Role of substratum stiffness in modulating genes associated with extracellular matrix and mechanotransducers YAP and TAZ. Invest Ophthalmol Vis Sci 54: 378-386, 2013.

20. Dupont S, Morsut L, Aragona M,Enzo E, Giulitti S, Cordenonsi M, Zanconato F, Le Digabel J, Forcato M, Bicciato S, et al: Role of YAP/TAZ in mechanotransduction. Nature 474: 179-183, 2011.

21. Li C, Chen J, Lu B, Shi Z, Wang H, Zhang B, Zhao K, Qi W, Bao J and Wang Y: Molecular switch role of Akt in Polygonatum odoratum lectin-induced apoptosis and autophagy in human non-small cell lung cancer A549 cells. PLoS One 9: e101526, 2014.

22. Huang G, Lv J, Li T, Huai G, Li X, Xiang S, Wang L, Qin Z, Pang J, Zou B, et al: Notoginsenoside R1 ameliorates podocyte injury in rats with diabetic nephropathy by activating the PI3K/Akt signaling pathway. Int J Mol Med 38: 1179-1189, 2016.

23. Wang Y, Liu Y, Wang H, Li C, Qi P and Bao J: Agaricus bisporus lectins mediates islet $\beta$-cell proliferation through regulation of cell cycle proteins. Exp Biol Med (Maywood) 237: 287-296, 2012

24. Wang Y, Wang H, Liu Y, Li C, Qi P and Bao J: Antihyperglycemic effect of ginsenoside $\mathrm{Rh} 2$ by inducing islet $\beta$-cell regeneration in mice. Horm Metab Res 44: 33-40, 2012.

25. Schmittgen TD and Livak KJ: Analyzing real-time PCR data by the comparative C(T) method. Nat Protoc 3: 1101-1108, 2008.

26. Perkins TW, Alvarado JA, Polansky JR, Stilwell L, Maglio M and Juster R: Trabecular meshwork cells grown on filters. Conductivity and cytochalasin effects. Invest Ophthalmol Vis Sci 29: 1836-1846, 1988. 
27. Thomasy SM, Morgan JT, Wood JA, Murphy CJ and Russell P: Substratum stiffness and latrunculin B modulate the gene expression of the mechanotransducers YAP and TAZ in human trabecular meshwork cells. Exp Eye Res 113: 66-73, 2013.

28. Rozsa FW, Reed DM, Scott KM, Pawar H, Moroi SE, Kijek TG, Krafchak CM, Othman MI, Vollrath D, Elner VM, et al: Gene expression profile of human trabecular meshwork cells in response to long-term dexamethasone exposure. Mol Vis 12: 125-141, 2006.

29. Steely HT, Browder SL, Julian MB, Miggans ST, Wilson KL and Clark AF: The effects of dexamethasone on fibronectin expression in cultured human trabecular meshwork cells. Invest Ophthalmol Vis Sci 33: 2242-2250, 1992.

30. Filla MS, Clark R and Peters DM: A syndecan-4 binding peptide derived from laminin 5 uses a novel PKCe pathway to induce cross-linked actin network (CLAN) formation in human trabecular meshwork (HTM) cells. Exp Cell Res 327: 171-182, 2014.

31. Clark AF, Miggans ST, Wilson K, Browder S and McCartney MD Cytoskeletal changes in cultured human glaucoma trabecular meshwork cells. J Glaucoma 4: 183-188, 1995.

32. Zhou L, Li Y and Yue BY: Glucocorticoid effects on extracellular matrix proteins and integrins in bovine trabecular meshwork cells in relation to glaucoma. Int J Mol Med 1: 339-346, 1998.

33. Peng J, Feng XY, Ye ZM, Luo Q, Cheng YL, Wu ZZ, Lei CT and Gong B: Effects of dexamethasone and HA1077 on actin cytoskeleton and $\beta$-catenin in cultured human trabecular meshwork cells. Int J Ophthalmol 9: 1376-1380, 2016.

34. Lange AW, Sridharan A, Xu Y, Stripp BR, Perl AK and Whitsett JA: Hippo/Yap signaling controls epithelial progenitor cell proliferation and differentiation in the embryonic and adult lung. J Mol Cell Biol 7: 35-47, 2015.

35. Morgan JT, Murphy CJ and Russell P: What do mechanotransduction, Hippo, Wnt, and TGF $\beta$ have in common? YAP and TAZ as key orchestrating molecules in ocular health and disease. Exp Eye Res 115: 1-12, 2013.

36. Filla MS, Schwinn MK, Sheibani N, Kaufman PL and Peters DM: Regulation of cross-linked actin network (CLAN) formation in human trabecular meshwork (HTM) cells by convergence of distinct beta 1 and beta 3 integrin pathways. Invest Ophthalmol Vis Sci 50: 5723-5731, 2009.

37. Rao PV, Deng PF, Kumar J and Epstein DL: Modulation of aqueous humor outflow facility by the Rho kinase-specific inhibitor Y-27632. Invest Ophthalmol Vis Sci 42: 1029-1037, 2001.

38. Sit AJ and Liu JH: Pathophysiology of glaucoma and continuous measurements of intraocular pressure. Mol Cell Biomech 6: 57-69, 2009

39. McKee CT, Wood JA, Shah NM, Fischer ME, Reilly CM, Murphy CJ and Russell P: The effect of biophysical attributes of the ocular trabecular meshwork associated with glaucoma on the cell response to therapeutic agents. Biomaterials 32: 2417-2423, 2011.
40. Wood JA, McKee CT, Thomasy SM, Fischer ME, Shah NM, Murphy CJ and Russell P: Substratum compliance regulates human trabecular meshwork cell behaviors and response to latrunculin B. Invest Ophthalmol Vis Sci 52: 9298-9303, 2011.

41. Wood JA, Shah NM, McKee CT, Hughbanks ML, Liliensiek SJ, Russell P and Murphy CJ: The role of substratum compliance of hydrogels on vascular endothelial cell behavior. Biomaterials 32: 5056-5064, 2011.

42. Chew SY and Low WC: Scaffold-based approach to direct stem cell neural and cardiovascular differentiation: an analysis of physical and biochemical effects. J Biomed Mater Res A 97: 355-374, 2011.

43. Han H, Wecker T, Grehn F and Schlunck G: Elasticity-dependent modulation of TGF- $\beta$ responses in human trabecular meshwork cells. Invest Ophthalmol Vis Sci 52: 2889-2896, 2011.

44. Zhao B, Li L, Lei Q and Guan KL: The Hippo-YAP pathway in organ size control and tumorigenesis: an updated version. Genes Dev 24: 862-874, 2010.

45. Wordinger RJ and Clark AF: Effects of glucocorticoids on the trabecular meshwork: towards a better understanding of glaucoma. Prog Retin Eye Res 18: 629-667, 1999.

46. Morrison DK: The 14-3-3 proteins: integrators of diverse signaling cues that impact cell fate and cancer development. Trends Cell Biol 19: 16-23, 2009.

47. Junglas B, Kuespert S, Seleem AA, Struller T, Ullmann S, Bösl M, Bosserhoff A, Köstler J, Wagner R, Tamm ER, et al: Connective tissue growth factor causes glaucoma by modifying the actin cytoskeleton of the trabecular meshwork. Am J Pathol 180: 2386-2403, 2012.

48. Tovar-Vidales T, Roque R, Clark AF and Wordinger RJ: Tissue transglutaminase expression and activity in normal and glaucomatous human trabecular meshwork cells and tissues. Invest Ophthalmol Vis Sci 49: 622-628, 2008

49. Mao W, Millar JC, Wang WH, Silverman SM, Liu Y, Wordinger RJ, Rubin JS, Pang IH and Clark AF: Existence of the canonical Wnt signaling pathway in the human trabecular meshwork. Invest Ophthalmol Vis Sci 53: 7043-7051, 2012.

50. Varelas X, Miller BW, Sopko R, Song S, Gregorieff A, Fellouse FA, Sakuma R, Pawson T, Hunziker W, McNeill H, et al: The Hippo pathway regulates Wnt/beta-catenin signaling. Dev Cell 18: 579-591, 2010

This work is licensed under a Creative Commons Attribution-NonCommercial-NoDerivatives 4.0 International (CC BY-NC-ND 4.0) License. 